\title{
Inflationary models inducing non-Gaussian metric fluctuations
}

\author{
Francis Bernardeau \\ Service de Physique Théorique, CEA/DSM/SPhT, \\ Unité de recherche associée au CNRS, CEA/Saclay 91191 Gif-sur-Yvette cédex \\ Jean-Philippe Uzan \\ Laboratoire de Physique Théorique, CNRS-UMR 862\%, \\ Bât. 210, Université Paris XI, F-91405 Orsay Cedex, France, \\ Institut d'Astrophysique de Paris, GReCO, CNRS-FRE 2435, 98 bis, Bd Arago, 75014 Paris, France.
}

(Dated: November 21, 2018)

\begin{abstract}
We construct explicit models of multi-field inflation in which the primordial metric fluctuations do not necessarily obey Gaussian statistics. These models are realizations of mechanisms in which non-Gaussianity is first generated by a light scalar field and then transferred into curvature fluctuations. The probability distribution functions of the metric perturbation at the end of inflation are computed. This provides a guideline for designing strategies to search for non-Gaussian signals in future CMB and large scale structure surveys.
\end{abstract}

Inflation generically predicts Gaussian initial metric fluctuations with an almost scale invariant power spectrum [1]. It is interesting to determine how general this property is. With the advent of large scale structure and CMB surveys it will be possible to test in details whether the initial conditions were Gaussian or not and in the latter case how they can be characterized. At the moment, no non-Gaussian signal has been detected in CMB data [2 but the number of modes that can be probed is still small. In large-scale structure surveys the number of independent modes that are observed is large but the difficulty arises from the non-linear gravitational dynamics [3] which can shadow the primordial nonGaussianity [1]. Having at our disposal models of inflation in which non-Gaussian adiabatic metric fluctuations are generated can then serve as a guideline for designing strategies for detecting primordial non-Gaussianities.

In single field inflation, the slow-roll conditions, if valid throughout the period during which the seeds of the large scale structures are generated, prevent the generation of observable primordial non-Gaussianities. The reason is that the potential needs to be both flat enough for the fluctuations to develop and steep enough for the nonlinearity to be significant. Note however that starting from a non-vacuum initial state [5] or allowing for sharp features in the potential [6] could lead to primordial nonGaussianities. In those cases these features will be localized in a narrow band of wavelengths and will affect the shape of the density fluctuation power spectrum.

The situation is richer when more than one light scalar field are present during inflation. In multi-field inflation models, a mixture of adiabatic and isocurvature fluctuations [7], that can be correlated or not [8], are generically produced. It opens the door to the generation of primordial non-Gaussianity [9] because the non-linear couplings can be much stronger in the isocurvature direction than in the (inflaton) adiabatic direction [9, 10]. When the fields are coupled, exchange between isocurvature and adiabatic modes can be important [11] which makes it possible to generate non-Gaussianity on a large band of wavelengths while preserving an adiabatic slow-roll type primordial power spectrum [12].

In a previous study [12], we have presented the building blocks for such models. It is based on the generation of non-Gaussian isocurvature fluctuations which are subsequently transferred to the adiabatic mode due to a bend in the classical inflaton trajectory. The necessary ingredients for such models are (i) the existence of a light scalar field that has non-linear coupling, typically quartic, and (ii) a coupling term in the potential leading to isocurvature-adiabatic mode mixing. This latter mechanism can be given a simple geometrical interpretation. On super Hubble scales, curvature fluctuations are induced by fluctuations of the total expansion in different parts of the universe. The relative duration of their inflationary phase can be affected if inflaton trajectory is bent. Fluctuations in the isocurvature modes generate a bundle of parallel trajectories along which inflation lasts longer or shorter depending on whether they lie in the outer or inner part of the bent (see Fig. 1 of Ref. [12]).

In Ref. 12], we characterized the expected statistical properties of the metric fluctuations that were shown to be the superposition of a Gaussian and a non-Gaussian contributions of the same variance. The relative weight of the two contributions is related to the bending angle in field space. We explicitly computed the probability distribution function (PDF) of the non-Gaussian contribution which appears to be described by a single new parameter, $\nu_{3}$ [see Eq. (6) below]. These results were obtained without the use of any explicit model. This letter is dedicated to the building of this kind of inflationary models involving either two or three fields. The shape of their PDF will be computed and compared to our previous analytical expression 12].

In general the curvature fluctuations, $\mathcal{R}$, can be com- 
puted on superhorizon scales as [13],

$$
\mathcal{R}=H \delta t .
$$

Isocurvature fluctuations can then induce curvature fluctuations only if in some way or another they can change the relative total number of $e$-fold.

This can actually be obtained in a very simple way in hybrid type model of inflation with three fields. In that case, one field, $\phi$, is the inflaton; the second field is a light scalar, $\chi$, with quartic coupling $\lambda \leq 1$ and the third field, $\sigma$, is coupled to the two others so that the end of inflation is triggered when $\sigma$ undergoes a phase transition. To be more explicit, let us examine the following model

$$
\begin{aligned}
V(\phi, \chi, \sigma)= & \frac{1}{2} m^{2} \phi^{2}+\frac{\lambda}{4 !} \chi^{4}+\frac{\mu}{2}\left(\sigma^{2}-\sigma_{0}^{2}\right)^{2} \\
& +\frac{g}{2} \sigma^{2}(\phi \cos \alpha+\chi \sin \alpha)^{2}
\end{aligned}
$$

where $\sigma_{0}$ is the final vev of $\sigma$ and $\alpha$ is the mixing angle between $\phi$ and $\chi$ in their coupling to $\sigma$.

Inflation ends when the effective mass of $\sigma$ vanishes, that is when

$$
g(\phi \cos \alpha+\chi \sin \alpha)^{2}-2 \mu \sigma_{0}^{2}=0 .
$$

The value of $\phi$ at the end of inflation is

$$
\phi_{\mathrm{end}} \equiv \frac{ \pm \sqrt{2 \mu / g} \sigma_{0}-\chi \sin \alpha}{\cos \alpha} .
$$

For $\phi>\phi_{\text {end }}, \sigma=0$ and the two fields evolve independently: $\phi$ drives the inflation while $\chi$ develops nonGaussianity. The amount of non-Gaussianity of $\chi$ then depends only on $\lambda$ and on the total number of $e$-fold between horizon crossing and the end of inflation.

When $\alpha$ is non-zero, fluctuations of $\chi$ induce curvature fluctuations because they change the time at which the phase transition occurs. Thus the $\chi$-induced curvature fluctuations read (assuming $H$ is basically constant during the inflationary period),

$$
\mathcal{R} \simeq H \delta t_{\text {end }} \simeq-\frac{3 H^{2}}{V_{, \phi}} \frac{\sin \alpha}{\cos \alpha} \chi .
$$

We present on Fig. 1 the result of a numerical investigation in which the super-Hubble evolution of the $\chi$ modes are explicitly computed. The Klein-Gordon equations for the two fields and the Friedmann equations are integrated for a set of initial conditions leading to a bundle of classical trajectories. The parameters of this example were taken to be $\lambda=10^{-2}$ and an evolution for a number of $e$-folds equal to $N_{e}=50$. The shape of the $\mathrm{PDF}$ is compared to our analytical expression [12],

$$
\begin{aligned}
P(\chi) \mathrm{d} \chi= & \sqrt{\frac{1}{2 \pi}\left|\frac{1-\chi^{2} \nu_{3}}{\left(1+\chi^{2} \nu_{3} / 3\right)^{3}}\right|} \times \\
& \exp \left[-\frac{3 \chi^{2}}{\left(6+2 \chi^{2} \nu_{3}\right) \sigma_{\chi}^{2}}\right] \frac{\mathrm{d} \chi}{\sigma_{\chi}} .
\end{aligned}
$$

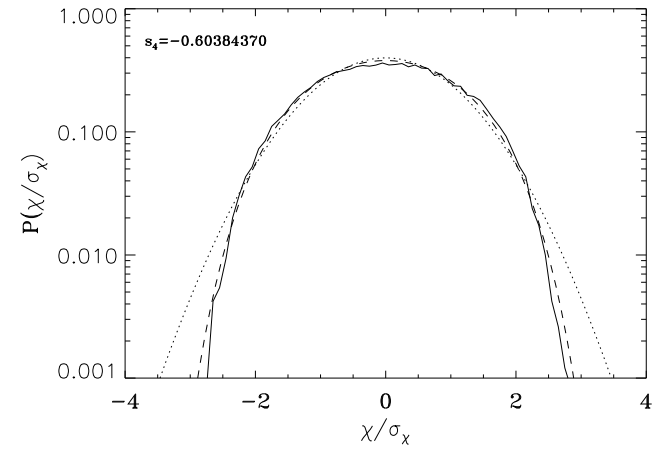

FIG. 1: The shape of the probability distribution function of the isocurvature modes in case of a quartic coupling, 2, (solid line) compared to the analytic PDF of Eq. (6) (dashed line) and to Gaussian distribution (dotted line). In this example, $\lambda=10^{-2}$ and $N_{e}=50$.

which depends only on the variance of $\chi$ and on the parameter $\nu_{3}$ quantifying the amount of non-Gaussianity. The variance is directly proportional to the value of the Hubble constant at horizon crossing, $H_{c}$. The parameter $\nu_{3}$ is proportional to $\lambda$ and is explicitly given by

$$
\nu_{3}=-\lambda N_{e} /\left(3 H_{c}^{2}\right) \approx-0.60 .
$$

On Fig. 1, the numerical results are compared to the expression (6) and to a Gaussian distribution of the same variance. It confirms that the analytic form (6) is a very good approximation of the PDF. The value of $\nu_{3}$ in this plot is 15] $\nu_{3}=-0.15 / \sigma_{\chi}^{2}$, which implies that the value of the kurtosis of distribution is

$$
s_{4} \equiv\left\langle\chi^{4}\right\rangle /\left\langle\chi^{2}\right\rangle^{2}-3=4 \nu_{3} \sigma_{\chi}^{2} .
$$

The curvature fluctuation PDF is related to the PDF of the field $\chi$. In the limit where the inflation ends abruptly the relation is straightforward, Eq. (5). It is more complex in general and depends on the details of the phase of inflation.

The parameters of the potential (2) are tuned, as usual, so that the amplitude of the fluctuations are compatible with the observations. No further fine-tunings are required for the initial conditions: whatever the initial value for the field $\chi$, it rolls down rapidly towards $\chi=0$ to follow the valley bottom, as described in the previous paragraphs.

The model (2) reads as a simple polynomial potential of order four but involves three fields. It is actually possible to build models involving only two fields. In this case, it is clear that the $\chi$ trajectory cannot be straight and has to bend. A possible explicit model can be obtained when the zero-mode trajectory is given by

$$
\chi_{0}(t)=\chi_{\infty} \tanh \left(\frac{\alpha \phi_{0}(t)}{\chi_{\infty}}\right)
$$

where $\alpha$ and $\chi_{\infty}$ are free parameters. A model to get such a trajectory can be constructed by considering a term of 
the form $\left(\chi-\chi_{0}\right)^{4}$ in the potential, as first advocated in Ref. [12], so that the potential might take the form

$V(\phi, \chi)=\frac{1}{2} m^{2} \phi^{2}+\frac{\lambda}{4 !}\left(\chi \cosh \left[\frac{\alpha \phi}{\chi_{\infty}}\right]-\chi_{\infty} \sinh \left[\frac{\alpha \phi}{\chi_{\infty}}\right]\right)^{4}$.

Although it gives the adequate trajectory (9) in field space, it is non-polynomial and does not induce significant metric non-Gaussianities. The reason is that the $\chi$ fluctuations are squashed as soon as the trajectory is bent, the bundle of trajectories being drastically focused much before any significant bending. This is a generic features of all models in which the bending of the trajectory is driven by the non-linear coupling term.

A way to construct a model in which the $\chi$ fluctuations are not squashed is to consider the potential

$$
\begin{aligned}
V(\phi, \chi)= & \frac{1}{2} m^{2}\left(\chi^{2}+\frac{\chi_{\infty}^{2}}{\alpha^{2}} \sinh ^{2}\left[\frac{\alpha \phi}{\chi_{\infty}}\right]\right)+ \\
& \frac{\lambda}{4 !}\left(\chi \cosh \left[\frac{\alpha \phi}{\chi_{\infty}}\right]-\chi_{\infty} \sinh \left[\frac{\alpha \phi}{\chi_{\infty}}\right]\right)^{4}
\end{aligned}
$$

Now, the zero mode trajectory $\left(\phi_{0}, \chi_{0}\right)$ is not determined by the non-linear coupling. At lowest order in the coupling parameter $\lambda$ and in the slow-roll regime, the KleinGordon equations for $\phi$ and $\chi$ can be integrated and lead to the trajectory (9).

In Fig. 2, we depict the behavior of the super-horizon $\phi$ and $\chi$ fluctuations (left panels) and the curvature fluctuations that are induced by the initial $\chi$ fluctuations (right panels). The parameters of the model for the simulation of Fig. 2 were chosen to be

$$
\begin{aligned}
& m=10^{-7} \cdot M_{\mathrm{Pl} .} \quad \alpha=1 \\
& \chi_{\infty}=1.5 M_{\mathrm{Pl} .} \quad \lambda=5 \times 10^{-5},
\end{aligned}
$$

so that it gives realistic fluctuation amplitudes and efold number. The initial conditions are set up at $\phi_{\text {init }}=4 M_{\mathrm{Pl} \text {. }}$. At this time the coupling constant is then about 0.15 and the number of $e$-folds during the inflationary period is about 72 . We integrate the KleinGordon equations and the Friedmann equation for the set of classical trajectories starting from different initial conditions around $\left(\phi_{\text {init }}, 0\right)$ Gaussian distributed with $\left\langle\left(\phi-\phi_{\text {init }}\right)^{2}\right\rangle \sim\left\langle\chi^{2}\right\rangle \sim H_{\text {init }}^{2}$.
As can be observed on the left panels the joint evolution of the $\phi$ and $\chi$ fluctuations is quite complex. This is due to the fact that the fluctuation distribution is shaped by the nonlinear couplings during the trajectory bend. The resulting curvature fluctuations can however be simply described. They are given by the sum of those induced by the $\phi$ fluctuations, $\mathcal{R}_{\phi}$, (Gaussian distributed) and those induced by the $\chi$ fluctuations, $\mathcal{R}_{\chi}$. Both distributions have approximately the same variance. For the parameters (12-13), our numerical computations show that actually the r.m.s. of the former is about $18 H_{c}$, the r.m.s. of the latter $15 H_{c}$. The right panels show the PDF shape of the non-Gaussian component of the curvature fluctuations, $\mathcal{R}_{\chi}$. They follow the distribution function given in Eq. (6) as long as $\left|s_{4}\right| \lesssim 0.7$ at least for less than $3 \sigma$ events. The complete PDF of the curvature fluctuations can then be obtained as the convolution of the PDFs of the two components, $\mathcal{R}_{\phi}$ and $\mathcal{R}_{\chi}$.

In this letter, we have introduced a series of multifield inflationary models leading to the generation of nonGaussian primordial curvature fluctuations. We have been able to build explicit models with either two or three scalar fields. In the case of two-field models, it is difficult to design a simple potential: it has to be constructed in such a way that the non-linear term does not damp the curvature fluctuations. In the case of three fields, we have shown that models as simple as polynomial potentials of order four work. In both cases we have illustrated our models by numerical computations and have shown that the analytic result (6) derived in Ref. [12] is a very good approximation to the primordial curvature fluctuation PDF when non-Gaussianities in the $\chi$ distribution remain modest. Let us emphasize that in the two-field models the potential has to be tuned but that this is not the case for the three-field model. Moreover, the initial conditions do not require to be fine-tuned, as explained before. This description of the primordial nonGaussianity is the starting point of further investigations on its various observational aspects, both for CMB and large scale structure surveys 14].
[1] A.D. Linde, Particle physics and inflationary cosmology, Harwood (Chur, Switzerland, 1990); A.R. Liddle and D.H. Lyth, Cosmological inflation and large-scale structure, Cambridge University Press (Cambridge, UK, 2000).

[2] H.B. Sandvik and J. Magueijo, astro-ph/0010395; G. Rocha et al., Phys. Rev. D 64 (2001) 06352; M.G. Santos et al., Phys. Rev. Lett. 88 (2002) 241302.

[3] F. Bernardeau, S. Colombi, E. Gaztañaga, and R. Scoccimaro, Phys. Rept. 367 (2002) 1.
[4] R. Durrer, R. Juszkiewicz, M. Kunz, and J.-P. Uzan, Phys. Rev. D 62 (2000) 021301(R).

[5] J. Martin, A. Riazuelo, and M. Sakellariadou, Phys. Rev. D 61 (2000) 083518.

[6] J. Lesgourgues, D. Polarski, and A.A. Starobinsky, Month. Not. R. Astron. Soc. 297 (1998) 769; A.A. Starobinsky, Grav. Cosmol. 4 (1998) 88.

[7] A.D. Linde, Phys. Lett. B 158 (1985) 375; L.A. Kofman, Phys. Lett. B 173 (1986) 400; D. Polarski and A.A. Starobinsky, Phys. Rev. D 50 (1994) 6123; J. García- 

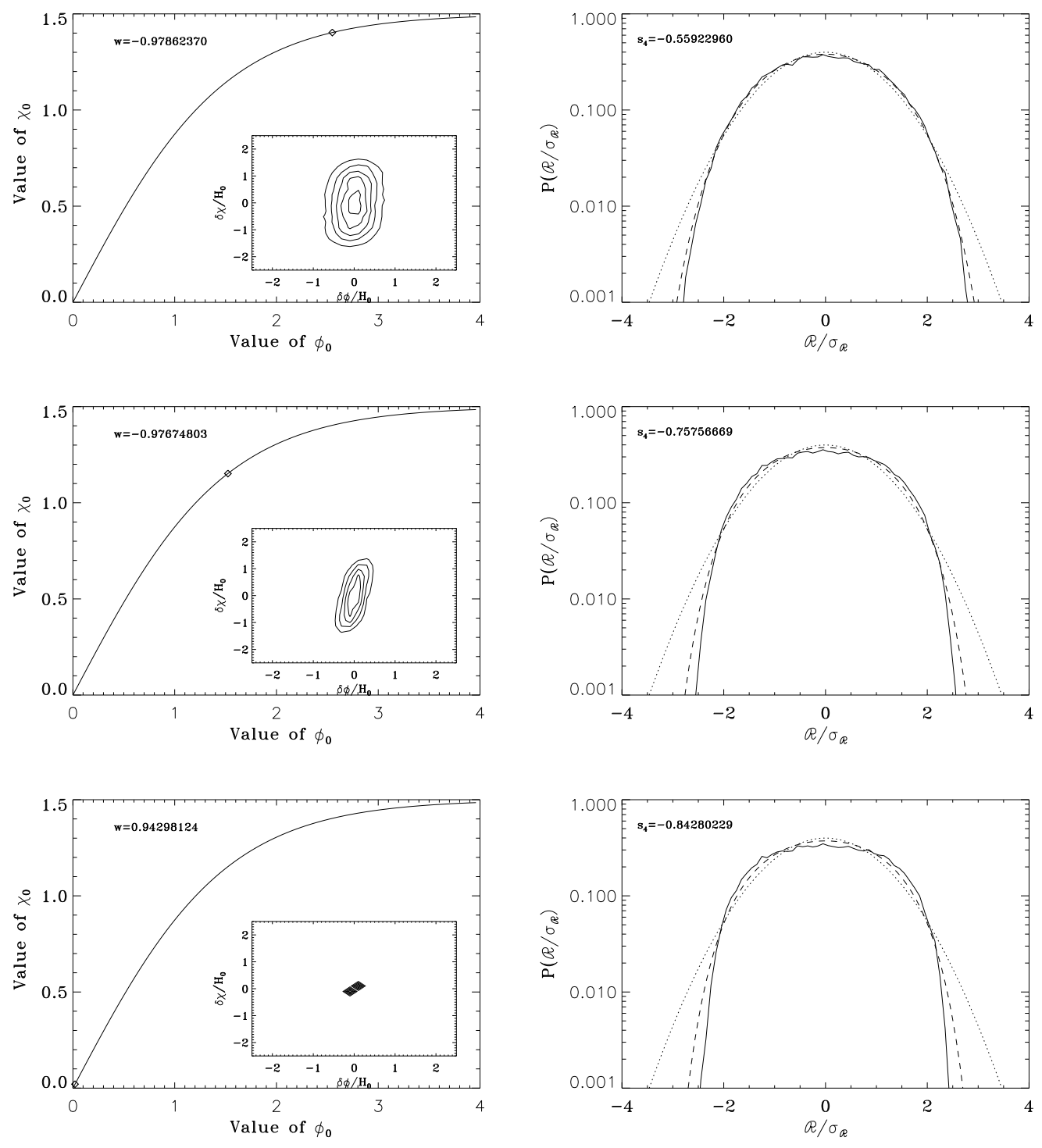

FIG. 2: PDF for the two-field model with potential (11) at different timestep with the parameters (12 13). The left panel describes the field trajectory and the shape of the wavepacket and the right panels compare the numerically obtained PDF (solid) to a Gaussian (dotted) and to the analytical PDF (6) (dash). $w$ is the effective equation of state parameter and $s_{4}$ is defined in Eq. (\$).

Bellido and D. Wands, Phys. Rev. D 53 (1996) 5437; V.F. Mukhanov and P.J. Steinhardt, Phys. Lett. B 422 (1998) 52 .

[8] D. Langlois, Phys. Rev. D 59 (1999) 123512; C. Gordon, D. Wands, B.A. Basset, and R. Maartens, Phys. Rev. D 63 (2000) 023506; J.H. Hwang and H. Noh, Phys. Lett. B 495 (2000) 277.

[9] A. Linde and V.F. Mukhanov, Phys. Rev. D 56 (1997) 535.

[10] D.S. Salopek, Phys. Rev. D 45 (1992) 1139; D.S. Salopek and J.R. Bond, Phys. Rev. D 42 (1990) 3936; D. La and P.J. Steinhardt, Phys. Rev. Lett. 62 (1989) 376; K. Ya- mamoto et al., Phys. Rev. D 46 (1992) 4206; D.H. Lyth, C. Ungarelli, and D. Wands, arXiv:astro-ph/0208055.

[11] N. Bartolo, S. Matarrese, and A. Riotto, Phys. Rev. D 64 (2001) 083514; ibid., Phys. Rev. D 65 (2002) 103505.

[12] F. Bernardeau and J.-P. Uzan, arXiv:hep-ph/0207295.

[13] A.R. Liddle and D.H. Lyth, Phys. Rept. 231 (1993) 1.

[14] F. Bernardeau, A. Riazuelo and J.-P. Uzan, in preparation.

[15] Note that the variance of the field value evolves due to the non-zero effective mass of $\chi$. 